\title{
Space Partitioning Evolutionary Many-Objective Opti- mization: Performance Analysis on MNK-Landscapes
}

\author{
Hernán Aguirre \\ Kiyoshi Tanaka
}

International Young Researcher Empowerment Center

Shinshu University, Faculty of Engineering, 4-17-1 Wakasato, Nagano, 380-8553 JAPAN

ahernaneshinshu-u.ac.jp

Shinshu University, Faculty of Engineering, 4-17-1 Wakasato, Nagano, 380-8553 JAPAN

ktanaka@shinshu-u.ac.jp

keywords: Space partitioning, many-objective evolutionary optimization, selection, epistasis, MNK-Landscapes

\begin{abstract}
Summary
This work proposes space partitioning, a new approach to evolutionary many-objective optimization. The proposed approach instantaneously partitions the objective space into subspaces and concurrently searches in each subspace. A partition strategy is used to define a schedule of subspace sampling, so that different subspaces can be emphasized at different generations. Space partitioning is implemented with adaptive $\epsilon$-ranking, a procedure that re-ranks solutions in each subspace giving selective advantage to a subset of well distributed solutions chosen from the set of solutions initially assigned rank-1 in the high dimensional objective space. Adaptation works to keep the actual number of rank-1 solutions in each subspace close to a desired number. The effects on performance of space partitioning are verified on MNK-Landscapes. Also, a comparison with two substitute distance assignment methods recently proposed for many-objective optimization is included.
\end{abstract}

\section{Introduction}

Multiobjective evolutionary algorithms (MOEAs) [Deb 01, Coello 02] optimize simultaneously two or more objective functions, aiming to find a set of trade-off solutions in a single run of the algorithm. Recently, there is a growing interest on applying MOEAs to solve manyobjective optimization problems, where the number of objective functions is four or more. However, high dimensionality imposes several difficulties and in general conventional MOEAs scale up poorly with the number of objectives, particularly Pareto based MOEAs [Purshouse 03, Aguirre 05, Hughes 05]. One of the difficulties is that the number of Pareto non-dominated solutions in the instantaneous population grows rapidly with the number of objectives [Aguirre 04]. In such case, multi-objective evolutionary algorithms that use Pareto-based selection cannot finerank solutions. This results in a weak selection pressure that translates into a severe deterioration of their search ability. The increased number of non-dominated solutions also poses a challenge to the density estimators that should favor an even distribution of solutions to balance selection pressure towards the different regions of objective space. Another difficulty is that the number of points required to represent accurately the Pareto front grows exponentially with the number of objectives. Current algorithms using population sizes in the order of hundreds can only generate very coarse approximations of the Pareto front. In addition, visualization, metrics to analyze performance of the algorithms, and an increased computational cost are other important issues in high dimensional spaces.

To cope with the challenges imposed by many-objective optimization new evolutionary algorithms are being proposed [Ishibuchi 08]. These new methods have focused mainly in the effectiveness of selection, dimensionality reduction, and the incorporation of user preferences. Some methods that focus on the effectiveness of selection try to improve Pareto-based selection by incorporating indicator functions or extensions of Pareto dominance [Zitzler 04, Emmerich 05, Corne 07, Kukkonen 07, Ishibuchi 07b, Koppen 07].Other methods use aggregation functions in the selection mechanism instead of the Pareto criteria [Ishibuchi 07a, Hughes 05].

Dimensionality reduction approaches try to reduce the number of objectives the evolutionary algorithm uses to solve the problem [Deb 06b, Brockhoff 07, López Jaimes 08]. Here the main challenge consists in how to determine a minimum set of objectives functions that preserves most of the characteristics of the original problem. When such reductions are possible, the (lower dimensional) problem 
becomes more amenable to the evolutionary algorithm and to the decision maker as well. However, even if dimensionality reduction is possible, there is no guarantee that the new dimension of the problem will be low enough to overcome the problems of weak selection pressure of conventional MOEAs.

Methods that focus on the incorporation of user preferences aim to provide the MOEA with a reference point so that it can concentrate its search on a small region of the Pareto front [Deb 06a]. Incorporation of user preferences is a very interesting and useful approach, however here the assumptions are that the user has a clear idea about the Pareto front and knows where to look for solutions. When such knowledge does not exist, we first need methods that can search effectively in many-objective spaces and generate an approximation of the Pareto front.

In practice, the optimization of real-world problems is not a single step process. Rather, it often consists of a series of steps in which the above mentioned approaches could be used to complement each other.

This work proposes space partitioning, a new approach to evolutionary many-objective optimization. The proposed approach instantaneously partitions the objective space into lower dimensional subspaces and concurrently searches in each subspace. Partitioning of the objective space into subspaces aims to instantaneously emphasize the search within lower dimensional objective spaces in order to find a good approximation of the true Pareto front in the original high dimensional space. The proposed approach uses a partition strategy to define a schedule of subspace sampling, so that different subspaces can be emphasized at different generations. Space partitioning can serve as a framework to improve the effectiveness of selection in high dimensional spaces, for dynamic dimensionality reduction, and to realize parallel implementations of many-objective optimization algorithms.

In this work we focus on space partitioning and concurrent subspace search as a way to improve the effectiveness of selection in high dimensional spaces. In this paper, we implement space partitioning with adaptive $\epsilon$-ranking [Aguirre 09b]. Adaptive $\epsilon$-ranking re-ranks solutions in each subspace, giving selective advantage to a subset of well distributed solutions chosen from the set of solutions initially assigned rank-1 in the original high dimensional objective space, so that selection can put more emphasizes in exploitation. Adaptation in the re-ranking procedure works to keep the actual number of rank-1 solutions in each subspace close to a desired number. The combination of space partitioning, partitioning strategy, and adaptive $\epsilon$ ranking allows to perform an effective search aiming to improve convergence and diversity of solutions on manyobjective problems. It should be mentioned that methods to divide the search space into regions, without reducing dimensionality of the space, have been proposed to parallelize evolutionary algorithms for two and three objective problems [Branke 04]. However, their extension to larger dimensions is not straight forward.

We verify the effects on performance of space partitioning by comparing adaptive $\epsilon$-ranking with and without space partitioning. We also examine three different partition strategies to investigate the influence of subspace sampling. In addition, we compare results of the space partitioning approach with two other many-objective optimizers [Aguirre 09c]. Namely, Subvector Dominance Assignment (SVDOM) and Epsilon Dominance Assignment (EPSDOM) [Koppen 07].

We test the algorithms on MNK-Landscapes [Aguirre 04, Aguirre 06]. MNK-Landscapes are scalable modeles of epistatic interactions for multi-objective combinatorial problems, useful to understand the fundamental search mechanisms of the algorithms and their scalability on subclasses of problems by varying the non-linear complexity $K$, number of objectives $M$, and size of the search space $2^{N}$ of the problem. In this work we analyze the scalability of the algorithms on subclasses of problems of increased complexity $(0 \leq K \leq 50)$ and increased dimensionality of the objective space $(4 \leq M \leq 10)$.

\section{Definitions}

Let us consider, without loss of generality, a maximization multiobjective problem with $M$ objectives:

$$
\text { maximize } \boldsymbol{f}(\boldsymbol{x})=\left(f_{1}(\boldsymbol{x}), f_{2}(\boldsymbol{x}), \cdots, f_{M}(\boldsymbol{x})\right)
$$

where $\boldsymbol{x} \in \mathcal{X}$ is a vector in the solution space $\mathcal{X}$, and $f_{1}, f_{2}, \cdots, f_{M}$ the $M$ objective functions to be optimized.

[Definition 1](Objective space $\phi)$ The objective space of the problem is determined by the set $\phi=\left\{f_{1}, f_{2}, \cdots, f_{M}\right\}$ of the $M$ objective functions to be optimized.

Pareto dominance and $\epsilon$-dominance [Laumanns 02] are two domination structures [Yu 74] of special relevance to this work and are defined as follows.

[Definition 2](Pareto dominance) A solution $\boldsymbol{x}$ is said to dominate other solution $y$ in the objective space $\phi$, denoted $\boldsymbol{f}(\boldsymbol{x}) \succeq \boldsymbol{f}(\boldsymbol{y})$, if:

$$
\begin{aligned}
& \forall f_{m} \in \phi \quad f_{m}(\boldsymbol{x}) \geq f_{m}(\boldsymbol{y}) \\
& \exists f_{m} \in \phi \quad f_{m}(\boldsymbol{x})>f_{m}(\boldsymbol{y}) .
\end{aligned}
$$

[Definition 3]( $\epsilon$-dominance) A solution $\boldsymbol{x}$ is said to $\epsilon$-dominate other solution $\boldsymbol{y}$ in the objective space $\phi$ for some $\epsilon>0.0$, denoted $\boldsymbol{f}(\boldsymbol{x}) \succeq^{\epsilon} \boldsymbol{f}(\boldsymbol{y})$, if:

$$
\begin{aligned}
& \forall f_{m} \in \phi \quad(1+\epsilon) f_{m}(\boldsymbol{x}) \geq f_{m}(\boldsymbol{y}) \quad \wedge \\
& \exists f_{m} \in \phi \quad(1+\epsilon) f_{m}(\boldsymbol{x})>f_{m}(\boldsymbol{y}) .
\end{aligned}
$$


Subspace, non-overlapping subspace, and space partition are important concepts we use to describe the proposed approach and are defined as follows.

[Definition 4](Subspace $\psi$ ) A subspace $\psi$ of $\phi$ is a lower dimension space that includes some of the functions in $\phi$, i.e. $\psi \subset \phi$.

[Definition 5](Non-overlapping subspaces) Two subspaces $\psi_{1} \subset \phi$ and $\psi_{2} \subset \phi$ are said to be non-overlapping if they have no common objectives, i.e. $\psi_{1} \cap \psi_{2}=\emptyset$.

[Definition 6](Space partition $\Psi_{N_{S}}$ ) An space $\phi$ is said to be partitioned into $N_{S}$ subspaces, denoted as $\Psi_{N_{S}}$, if all subspaces are non-overlapping and no objective function in $\phi$ is left unassigned to a subspace, i.e. $\Psi_{N_{S}}=$ $\left\{\psi_{1}, \psi_{2}, \cdots, \psi_{N_{S}} \mid \psi_{1} \cap \psi_{2} \cdots \cap \psi_{N_{S}}=\emptyset \wedge \psi_{1} \cup \psi_{2} \cdots \cup\right.$ $\left.\psi_{N_{S}}=\phi\right\}$.

The proposed method uses $\epsilon$-dominance to re-rank solutions within a subspace, taking into account only the objective functions assigned to the subspace. To distinguish $\epsilon$-dominance applied on a subspace from conventional $\epsilon$ dominance, subspace $\epsilon$-dominance is defined as follows.

[Definition 7](Subspace $\epsilon$-dominance) A solution $\boldsymbol{x}$ is said to $\epsilon$-dominate other solution $\boldsymbol{y}$ in the subspace $\psi$ for some $\epsilon>0.0$, denoted $\boldsymbol{f}(\boldsymbol{x}) \succeq_{\psi}^{\epsilon} \boldsymbol{f}(\boldsymbol{y})$, if:

$$
\begin{aligned}
& \forall f_{m} \in \psi \quad(1+\epsilon) f_{m}(\boldsymbol{x}) \geq f_{m}(\boldsymbol{y}) \quad \wedge \\
& \exists f_{m} \in \psi \quad(1+\epsilon) f_{m}(\boldsymbol{x})>f_{m}(\boldsymbol{y}) .
\end{aligned}
$$

\section{Method}

\section{$3 \cdot 1 \in \boldsymbol{R}-\mathrm{EMO}$}

In this section, we describe the proposed space partitioning with adaptive $\epsilon$-ranking method, called $\epsilon$ R-EMO for short. $\epsilon$ R-EMO first ranks solutions by Pareto dominance calculated in the original high-dimensional objective space. Then, it instantaneously partitions the objective space into subspaces, re-ranks solutions for each subspace using an adaptive subspace- $\epsilon$-ranking procedure, and concurrently performs one generation of the evolutionary search within each subspace. During the next cycle of the algorithm, parents and offspring from all subspaces will be joined together so that they will be ranked again in the original high dimensional objective space.

By partitioning the objective space into subspaces, we aim to instantaneously emphasize the search within lower dimensional objective spaces. At each generation, we don't search in all possible subspaces. Instead, we define a schedule of subspace sampling by using a partition strategy. Reranking of solutions by the adaptive subspace- $\epsilon$-ranking gives selective advantage to a subset of well distributed solutions chosen from the usually too many solutions assigned rank-1 in a high dimensional space, so that selec- tion can put more emphasizes in exploitation while balancing selection pressure towards the different regions of objective space. Adaptation in the re-ranking procedure works to keep the actual number of rank-1 solutions in each subspace close to a desired number. The combination of space partitioning, partitioning strategy, and adaptive subspace- $\epsilon$-ranking, aims to effectively search on manyobjective problems.

\section{Procedure $1 \epsilon$ R-EMO \\ Input: $N_{S}$, number of subspaces at each generation. $\alpha$, desired number of rank-1 solutions in each subspace (as a fraction of the entire parent population)}

Output: $\mathcal{F}_{1}$, set of Pareto non-dominated solutions

$$
\begin{aligned}
& \text { 1: } \mathcal{P} \leftarrow \emptyset, \mathcal{Q} \leftarrow \text { random } \\
& \text { // initialize parent } \mathcal{P} \text { and offspring } \mathcal{Q} \text { populations } \\
& \text { 2: } \epsilon_{1}, \epsilon_{2}, \cdots, \epsilon_{N_{S}} \leftarrow 0.0 \\
& \text { 3: } \Delta_{1}, \Delta_{2}, \cdots, \Delta_{N_{S}} \leftarrow \Delta_{0} \\
& \text { 4: repeat } \\
& \text { 5: } \quad \text { evaluation }(\mathcal{Q}, \phi) / / \phi=\left\{f_{1}, f_{2}, \cdots, f_{M}\right\} \\
& \text { 6: } \quad \mathcal{F} \leftarrow \text { non-domination-sorting }(\mathcal{P} \cup \mathcal{Q}) \\
& / / \mathcal{F}=\left\{\mathcal{F}_{i}\right\} \quad\left(i=1,2 \cdots, N_{F}\right) \\
& \text { 7: } \quad \text { crowding-distance }(\mathcal{F}) \\
& \text { 8: } \quad \Psi_{N_{S}} \leftarrow \text { space-partitioning }\left(\phi, N_{S}\right) \\
& / / \Psi_{N_{S}}=\left\{\psi_{1}, \psi_{2}, \cdots, \psi_{N_{S}}\right\} \\
& \text { 9: } \quad P \leftarrow \emptyset, Q \leftarrow \emptyset \\
& \text { 10: } \quad \text { for } s=1 \text { to } N_{S} \text { do } \\
& \text { 11: } \quad \mathcal{F}^{\epsilon_{s}} \leftarrow \text { subspace- } \epsilon \text {-ranking }\left(\psi_{s}, \epsilon_{s}, \mathcal{F}\right) \\
& / / \mathcal{F}^{\epsilon_{s}}=\left\{\mathcal{F}_{j}^{\epsilon_{s}}\right\} \quad\left(j=1,2 \cdots, N_{F}^{\epsilon_{s}}\right) \\
& \text { 12: } \quad \epsilon_{s}, \Delta_{s} \leftarrow \epsilon \text {-adaptation }\left(\epsilon_{s}, \Delta_{s}, \alpha,\left|\mathcal{F}_{1}^{\epsilon_{s}}\right|\right) \\
& \text { // adapt } \epsilon_{s} \text { and } \Delta_{s} \text { for the next generation } \\
& \text { 13: } \quad \mathcal{P}_{s} \leftarrow \operatorname{truncation}\left(\mathcal{F}^{\epsilon_{s}}\right) \\
& / /\left|\mathcal{P}_{s}\right|=|\mathcal{P}| / N_{S},\left|\mathcal{F}^{\epsilon_{s}}\right|=|\mathcal{P}|+|\mathcal{Q}| \\
& \text { 14: } \quad \mathcal{Q}_{s} \leftarrow \text { recombination and mutation }\left(\mathcal{P}_{s}\right) \\
& / /\left|\mathcal{Q}_{s}\right|=|\mathcal{Q}| / N_{S} \\
& \text { 15: } \quad \mathcal{P} \leftarrow \mathcal{P} \cup \mathcal{P}_{s}, \mathcal{Q} \leftarrow \mathcal{Q} \cup \mathcal{Q}_{s} \\
& \text { 16: end for } \\
& \text { 17: until termination criterion is met } \\
& \text { 18: return } \mathcal{F}_{1}
\end{aligned}
$$

$\epsilon$ R-EMO implemented in NSGA-II's framework [Deb 00] is illustrated in Procedure 1. In the following we explain in detail its distinctive features.

\subsection{Objective Space Partitioning}

In our approach, we partition the $M$ dimensional space $\phi=\left\{f_{1}, f_{2}, \cdots, f_{M}\right\}$ into $N_{S}$ non-overlapping subspaces $\Psi_{N_{S}}=\left\{\psi_{1}, \psi_{2}, \cdots, \psi_{N_{S}}\right\}$ (Procedure 1, line 8). All subspaces have the same dimension $M_{S}=M / N_{S}$ in case $r=$ $\left(M \bmod N_{S}\right)$ is zero. Otherwise, $r$ of the $N_{S}$ subspaces have dimension $M_{S}=\left\lfloor M / N_{S}\right\rfloor+1$ and the rest $M_{S}=$ 
Generation $\mathrm{t}$

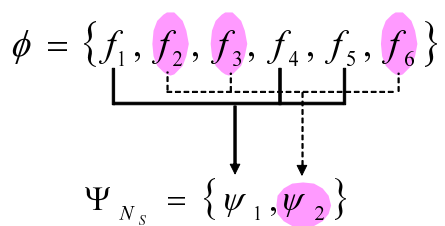

Generation $t+1$

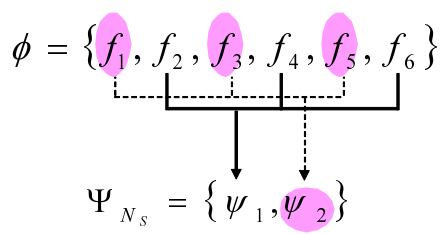

(a) random strategy
Generation t

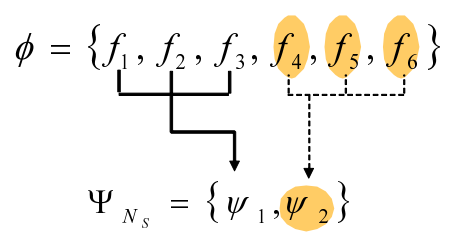

Generation $\mathrm{t}+1$

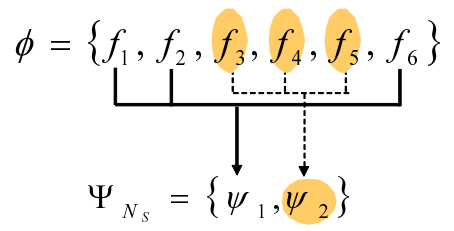

(b) shift strategy
Generation t

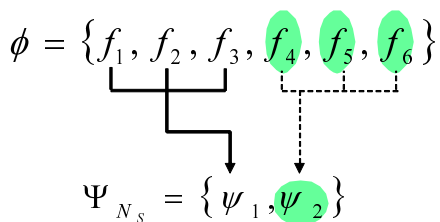

Generation $\mathrm{t}+1$

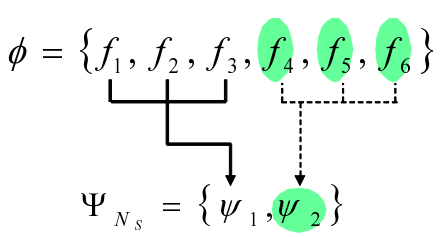

(c) fixed strategy

Fig. 1 Space partitioning strategies.

$\left\lfloor M / N_{S}\right\rfloor$. The number of all possible ways to partition $\phi$ into subspaces of dimension $M_{S}$ is given by $\frac{M !}{M_{S} !\left(M-M_{S}\right) !}$ and becomes very large with $M$. For example, for $M=$ $\{4,6,8,10\}$ objectives and $N_{S}=2$ subspaces there are $\{6,20,70,252\}$ ways to partition the space, respectively. In our approach, we don't explicitly search in all possible subspaces at each generation. Instead, we set $N_{S}$ to a small value and define a schedule of subspace sampling by using a partitioning strategy. We investigate three strategies to partition $\phi$. Namely, random, shift, and fixed partition strategies as illustrated in Figure 1 .

Random strategy randomly assigns objectives $f_{i} \in \phi$ to subspaces $\psi_{s} \in \Psi_{N_{S}}$. With this strategy, any possible $M_{S}$ dimensional subspace of $\phi$ could be formed. However, it does not seek to correlate the $s$-th subspace $\psi_{s}$ from generation $t$ to the next.

Shift strategy, at the first generation, assigns deterministically objectives $f_{i} \in \phi$ to subspaces $\psi_{s} \in \Psi_{N_{S}}$, so that objectives assigned to a given $\psi_{s}$ are ordered by objective index $i$. Then, in subsequent generations, the objective with highest index in the $s$-th subspace is shifted to the $\left((s+1) \bmod N_{S}\right)$-th subspace, $\forall \psi_{s} \in \Psi_{N_{S}}$. This strategy correlates the $s$-th subspace from generation $t$ to the next. In fact, subspace $\psi_{s}$ at generation $t$ overlaps with $\psi_{s}$ at generation $t-1$ in all but one objective. However, only $M$ of all possible $M_{S}$ dimensional subspaces of $\phi$ could be formed.

Fixed strategy assigns deterministically objectives $f_{i} \in$ $\phi$ to subspaces $\psi_{s} \in \Psi_{N_{S}}$ and keeps the same assignment throughout the generations. With this strategy only $N_{S}=$ $M / M_{S}$ subspaces of $\phi$ could be formed.

Note that when a subspace is created by one of the three partitioning strategies we do not linearly aggregate objective functions nor apply other transformation to the individual objectives. We just indicate which functions in the space $\phi$ will be include in the subspace $\psi_{s} \in \Psi_{N_{S}}$.

The above strategies would allow us to verify the impact of subspace sampling on the quality of solutions and the effect of subspace correlation from one generation to the next on the adaptation of $\epsilon$ for subspace- $\epsilon$-ranking .

\subsection{Adaptation of $\epsilon$}

$\epsilon$ R-EMO re-ranks solutions in each subspace by using a subspace- $\epsilon$-ranking procedure (Procedure 1, line 11) in which the number of rank-1 solutions depends on the value set to $\epsilon(\geq 0)$ and on the instantaneous distribution of solutions in objective space. The adaptive procedure adapts $\epsilon$ (Procedure 1, line 12) at each generation in order to keep in each subspace the actual number of rank-1 solutions close to a desired number specified by $\alpha \times|\mathcal{P}|$, where $\alpha$ is a parameter in the range $[0.0,1.0]$ set by the user and $|\mathcal{P}|$ is the size of the entire parent population. In addition to adapting $\epsilon$, the step of adaptation $\Delta$ is also adapted to properly follow the dynamics of the evolutionary process on a given problem. Basically, if the number of solutions assigned rank- 1 by subspace- $\epsilon$-ranking is above the desired number $\alpha \times|\mathcal{P}|$ then $\Delta$ is updated by $\Delta \leftarrow \min \left(\Delta \times 2.0, \Delta_{\max }\right)$ and $\epsilon$ by $\epsilon \leftarrow \epsilon+\Delta$. Otherwise, $\Delta \leftarrow \max \left(\Delta \times 0.5, \Delta_{\text {min }}\right)$ and $\epsilon \leftarrow \epsilon-\Delta$, where the range of the step of adaptation is $\left[\Delta_{\min }, \Delta_{\max }\right]$.

$\epsilon \mathrm{R}$-EMO adapts one $\epsilon_{s}$ with its corresponding step of adaptation $\Delta_{s}$ for each one of the $N_{S}$ subspaces $\psi_{s}$, instead of using one $\epsilon$ for all subspaces. Note that the actual combination of objectives that define $\psi_{s}$ change from one generation to the next, depending on the partition strategy. So, adaptation of $\epsilon_{s}$ reacts to the characteristics of the different instantaneous subspaces (actual combinations of $f_{i}$ ) assigned to $\psi_{s}$. Since the dimension of the subspace is strongly correlated to the value of $\epsilon$ that renders the desired number of rank-1 solutions $\alpha \times|P|$, when the space 
$\phi$ is partitioned we make sure that the dimension of the subspace $\psi_{s}$ remains the same throughout the generations.

\section{$3 \cdot 4$ Subspace- $\epsilon$-ranking}

Subspace- $\epsilon$-ranking fine grains ranking of solutions initially ranked by Pareto dominance in the objective space $\phi$, using a randomized $\epsilon$-sampling procedure in the subspace $\psi \subset \phi$ that favors a good distribution of solutions based on dominance regions wider than conventional Pareto dominance. Subspace- $\epsilon$-ranking extends $\epsilon$-ranking [Aguirre 08], where $\epsilon$-sampling acts on $\phi$ instead of $\psi$. In the following, we first explain $\epsilon$-sampling and then subspace- $\epsilon$ ranking.

$\epsilon$-sampling assumes that there is a set of equally ranked solutions from which a subset should be chosen to give them selective advantage in order to proceed further with the evolutionary search. That is, $\epsilon$-sampling acts as a decision making procedure, not to find a final solution, but to help selection of the evolutionary algorithm. Hence, the sampling heuristic must reflect criteria that favor an effective search. Here, the sample of solutions to be given selective advantage are obtained with the following criteria,

- Extreme solutions are always part of the sample.

- Each (not extreme) sampled solution is the sole sampled representative of its area of influence. The area of influence of the sampled solutions is determined by a domination region wider than Pareto dominance, i.e. $\epsilon$-dominance.

- Sampling of (not extreme) solutions follows a random schedule.

The first criterion tries to push the search towards the optimum values of each fitness function, aiming to find non-dominated solutions in a wide area of objective space. The second criterion assures that only one solution in a given zone of objective space is given rank-1, trying to distribute the search effort more or less uniformly among the different zones represented in the actual population. The third criterion dynamically establishes the zones that are represented in the sample. Also, in the case that there are several solutions within each zone, it increases the likelihood that the sampled solutions that will be given higher rank are different from one generation to the next, increasing the possibility of exploring wider areas of objective and variable space.

Procedure 2 illustrates $\epsilon$-sampling algorithm. Let us denote $\mathcal{A}$ the set of solutions that have been assigned the same rank based on conventional Pareto dominance, for example by applying non-domination sorting [Deb 00]. $\epsilon$ sampling returns the sampled solutions $\mathcal{S} \subset \mathcal{A}$ that will be given selective advantage as well as the set of solutions $\mathcal{D}^{\epsilon}$ to be demoted. See that extreme solutions are the first to be assigned to the sample $\mathcal{S}$ (lines 1,2). Then, one by one, solutions are randomly chosen and included in $\mathcal{S}$ (lines 4-6), whereas solutions that lie in the wider domination region of the randomly picked solution are assigned to $\mathcal{D}^{\epsilon}$ (lines 7,8). Note that subspace $\epsilon$-dominance $\boldsymbol{f}(z) \succeq_{\psi}^{\epsilon} \boldsymbol{f}(y)$ is used. Figure 2 (a) illustrates the application of $\epsilon$-sampling on the set of solutions $\mathcal{A}=\mathcal{F}_{1}$. The numbers close to the solutions represents the random schedule in which solutions are sampled ( 0 means extreme solutions, which are all selected at once).

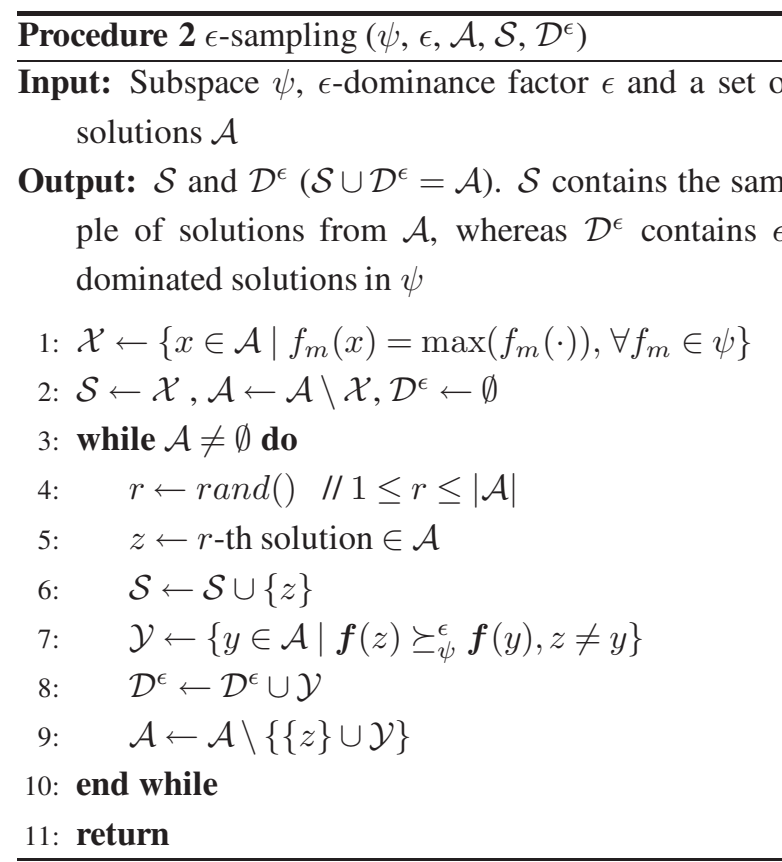

The $\epsilon$-sampling procedure works on a set of equally ranked solutions, however within a population there could be several sets of such solutions (each set with a different rank). To re-rank all possible sets of equally ranked solutions, we use subspace- $\epsilon$-ranking.

Subspace- $\epsilon$-ranking is applied at each generation for each subspace after non-domination sorting to reclassify the sets $\mathcal{F}_{i}\left(i=1, \cdots, N_{F}\right)$. Procedure 3 describes subspace- $\epsilon$ ranking algorithm. See that the reclassified sets $\mathcal{F}_{j}^{\epsilon_{s}}(j=$ $1, \cdots, N_{F}^{\epsilon_{s}}$ ) now contains only the sample of solutions $\mathcal{S} \subset$ $\mathcal{F}_{i}$ found by $\epsilon$-sampling (lines 8,9 ). Also, see that solutions $\mathcal{D}^{\epsilon}$, which are not part of the sample (line 8) are demoted by joining them with solutions of a lower ranked set in the next iteration of the loop (line 4 ). Thus, $\mathcal{F}_{1}^{\epsilon_{s}}$ contains some of the solutions initially ranked first, but $\mathcal{F}_{j}^{\epsilon_{s}}$, $j>1$, can contain solutions that initially were assigned to sets with different ranks. This gives chance to lateral diversity present in the initial ranking of solutions and can punish highly crowded solutions even if they are initially ranked first by conventional Pareto dominance. 


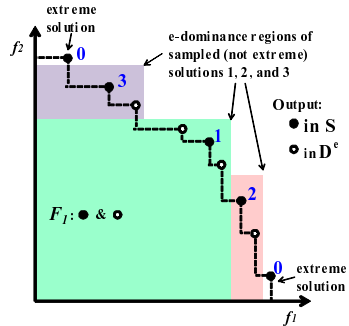

(a) $\mathcal{F}_{1}$

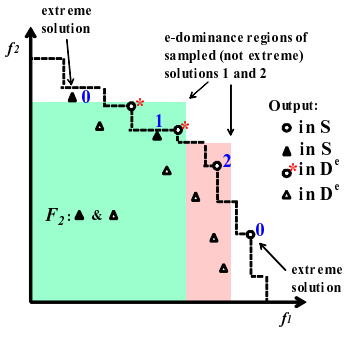

(b) $\mathcal{F}_{2} \cup \mathcal{D}_{\mathcal{F}_{1}}^{\epsilon}$
Fig. $2 \epsilon$-sampling on (a) the set of solutions initially ranked first and (b) on the set of solutions initially ranked second joined with solutions demoted from the first set.

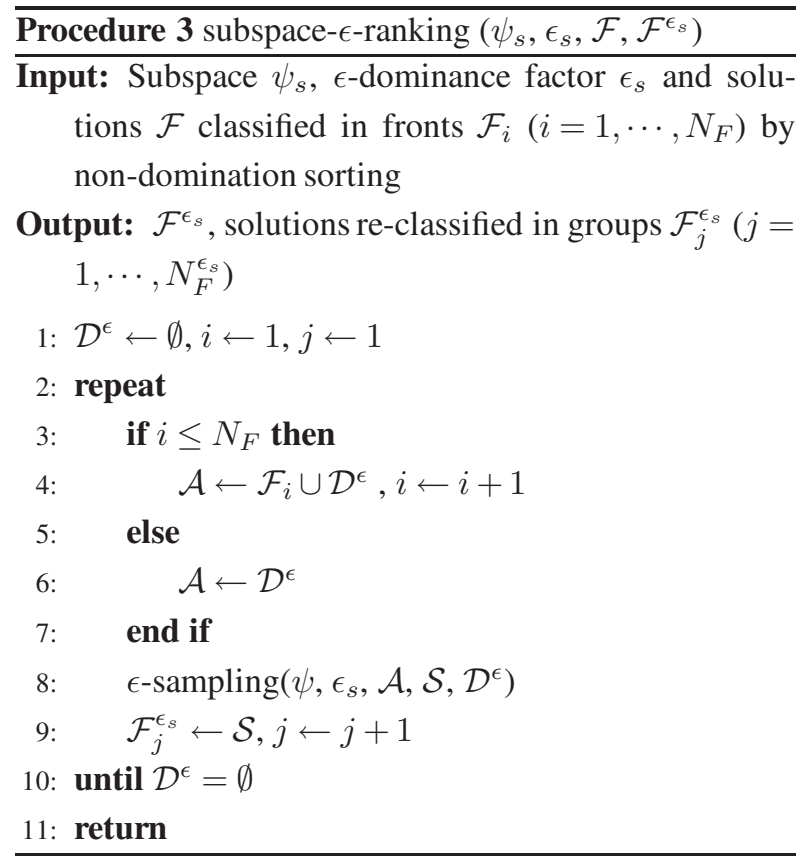

Figure 2 illustrates $\epsilon$-ranking calling on $\epsilon$-sampling to re-rank the set $\mathcal{F}_{1}$ of solutions initially ranked first and the set $\mathcal{F}_{2}$ of solutions ranked second joined with the demoted solutions $\mathcal{D}^{\epsilon}$ from $\mathcal{F}_{1}$. The example illustrates the application of $\epsilon$-sampling to a 2 dimensional objective space $\phi$. When $\epsilon$-sampling is applied to a subspace $\psi \subset \phi$, the non-dominated solutions in $\phi$ projected in the subspace $\psi$ (assuming a 2 dimensional subspace) would look similar to Figure 2 (b). $\epsilon$-sampling will be applied to all projected solutions.

\section{Multiobjective MNK-Landscapes}

In this work we use multiobjective MNK-Landscapes as test problems. A multiobjective MNK-Landscape [Aguirre 04, Aguirre 06] is defined as a vector function mapping binary strings into real numbers $\boldsymbol{f}(\cdot)=\left(f_{1}(\cdot), f_{2}(\cdot), \cdots\right.$, $\left.f_{M}(\cdot)\right): \mathcal{B}^{N} \rightarrow \Re^{M}$, where $M$ is the number of objectives, $f_{i}(\cdot)$ is the $i$-th objective function, $\mathcal{B}=\{0,1\}$, and $N$ is the bit string length. $\boldsymbol{K}=\left\{K_{1}, \cdots, K_{M}\right\}$ is a set of integers where $K_{i}(i=1,2, \cdots, M)$ is the number of bits in the string that epistatically interact with each bit in the $i$-th landscape. Each $f_{i}(\cdot)$ can be expressed as an average of $N$ functions as follows

$$
f_{i}(\boldsymbol{x})=\frac{1}{N} \sum_{j=1}^{N} f_{i, j}\left(x_{j}, z_{1}^{(i, j)}, z_{2}^{(i, j)}, \cdots, z_{K_{i}}^{(i, j)}\right)
$$

where $f_{i, j}: \mathcal{B}^{K_{i}+1} \rightarrow \Re$ gives the fitness contribution of bit $x_{j}$ to $f_{i}(\cdot)$, and $z_{1}^{(i, j)}, z_{2}^{(i, j)}, \cdots, z_{K_{i}}^{(i, j)}$ are the $K_{i}$ bits interacting with bit $x_{j}$ in the string $\boldsymbol{x}$. The fitness contribution $f_{i, j}$ of bit $x_{j}$ is a number between $[0.0,1.0]$ drawn from a uniform distribution. Thus, each $f_{i}(\cdot)$ is a nonlinear function of $\boldsymbol{x}$ expressed by a NK-Landscape model of epistatic interactions [Kauffman 93].

For a given $N$, we can tune the ruggedness of the fitness function $f_{i}(\cdot)$ of the $i$-th objective by varying $K_{i}$. In the limits, $K_{i}=0$ corresponds to a model in which there are no epistatic interactions and the fitness contribution from each bit value is simply additive, which yields a single peaked smooth $i$-th fitness landscape. On the opposite extreme, $K_{i}=N-1$ corresponds to a model in which each bit value is epistatically affected by all the remaining bit values yielding a maximally rugged fully random $i$-th fitness landscape. Varying $K_{i}$ from 0 to $N-1$ gives a family of increasingly rugged multi-peaked landscapes.

In addition to $\mathrm{M}, \mathrm{N}$, and $\boldsymbol{K}$, it is also possible to specify the epistatic pattern between bit $x_{j}$ and the $K_{i}$ other interacting bits. That is, the distribution $D_{i}=\{$ random, nearest neighbor $\}$ of $K_{i}$ bits among $N$. Thus, $M, N$, $\boldsymbol{K}=\left\{K_{1}, K_{2}, \cdots, K_{M}\right\}$, and $\boldsymbol{D}=\left\{D_{1}, D_{2}, \cdots, D_{M}\right\}$, completely specify a multiobjective MNK-Landscape.

MNK-Landscapes are easy to construct and can scale to any number of objectives $M$, number of bits $N$, and number of epistatic interactions $K_{i}$, allowing the creation of sub-classes of combinatorial non-linear problems for discrete search spaces. By varying these parameters we can analyze the properties of the multiobjective landscapes and study the effects of the number of objectives, size of the search space, intensity of epistatic interactions, and epistatic pattern on the performance of multiobjective optimization algorithms on combinatorial discrete search spaces.

\section{Performance Measures and Parameters}

\section{$5 \cdot 1$ Performance measures}

In this work, we use the hypervolume $\mathcal{H}$, the set coverage $\mathcal{C}$, and the normalized maximum spread $\mathcal{D}$ measures [Zitzler 99] to evaluate and compare the performance of the algorithms. The measure $\mathcal{H}$ calculates the volume of the $M$-dimensional region in objective space enclosed by 
a set of non-dominated solutions and a dominated reference point. Let $\mathcal{A}$ be a set of non-dominated solutions. The hypervolume of $\mathcal{A}$ can be expressed as

$$
\mathcal{H}(\mathcal{A})=\lambda\left(\cup_{i=1}^{|\mathcal{A}|} \mathcal{V}_{i}\right)
$$

where $\lambda$ is the Lebesgue measure, and $\mathcal{V}_{i}$ is the hypervolume rendered by the point $x_{i} \in \mathcal{A}$ and the reference point. In this work, the reference point is set to $[0.0, \cdots, 0.0]$. Given two sets of non-dominated solutions $\mathcal{A}$ and $\mathcal{B}$, if $\mathcal{H}(\mathcal{A})>\mathcal{H}(\mathcal{B})$ then set $\mathcal{A}$ can be considered better on convergence and/or diversity of solutions. To calculate $\mathcal{H}$, we use Fonseca et al. [Fonseca 06] algorithm, which significantly reduces computational time.

The set coverage $\mathcal{C}$ measure [Zitzler 99] provides complementary information on convergence. Let us denote $\mathcal{A}$ and $\mathcal{B}$ the sets of non-dominated solutions found by two algorithms. $\mathcal{C}(\mathcal{A}, \mathcal{B})$ gives the fraction of solutions in $\mathcal{B}$ that are dominated at least by one solution in $\mathcal{A}$. More formally,

$$
\mathcal{C}(\mathcal{A}, \mathcal{B})=\frac{|\{\boldsymbol{b} \in \mathcal{B} \mid \exists \boldsymbol{a} \in \mathcal{A}: \boldsymbol{f}(\boldsymbol{a}) \succeq \boldsymbol{f}(\boldsymbol{b})\}|}{|\mathcal{B}|} .
$$

$\mathcal{C}(\mathcal{A}, \mathcal{B})=1.0$ indicates that all solutions in $\mathcal{B}$ are dominated by solutions in $\mathcal{A}$, whereas $\mathcal{C}(\mathcal{A}, \mathcal{B})=0.0$ indicates that no solution in $\mathcal{B}$ is dominated by solutions in $\mathcal{A}$. Since usually $\mathcal{C}(\mathcal{A}, \mathcal{B})+\mathcal{C}(\mathcal{B}, \mathcal{A}) \neq 1.0$, both $\mathcal{C}(\mathcal{A}, \mathcal{B})$ and $\mathcal{C}(\mathcal{B}, \mathcal{A})$ are required to understand the degree to which solutions of one set dominate solutions of the other set.

The maximum spread $\mathcal{D}$ [Zitzler 99] measures the length of the diagonal of a hyperbox formed by the extreme function values observed in the non-dominated set. A normalized version of $\mathcal{D}$ [Deb 01] is expressed by

$$
\mathcal{D}=\sqrt{\frac{1}{M} \sum_{m=1}^{M}\left(\frac{\max _{i=1}^{\left|\mathcal{F}_{1}\right|} f_{m}^{i}-\min _{i=1}^{\left|\mathcal{F}_{1}\right|} f_{m}^{i}}{f_{m}^{\max }-f_{m}^{\min }}\right)^{2}}
$$

Here $\mathcal{F}_{1}$ is the set of non-dominated solutions, and $f_{m}^{\max }$ and $f_{m}^{m i n}$ are the maximum and minimum value of the $m$ th objective. A larger value of $\mathcal{D}$ indicates that a widely spread set of solutions is obtained. In this work we set $f_{m}^{\max }=1.0$ and $f_{m}^{\min }=0.0$, the maximum and minimum possible values in MNK-Landscapes.

\section{$5 \cdot 2$ Parameters}

The performance of the algorithms is verified on MNKLandscapes with $4 \leq M \leq 10$ objectives, $N=100$ bits, number of epistatic interactions $K=\{0,1,3,5,7,10,15$, $25,35,50\}\left(K_{1}, \cdots, K_{M}=K\right)$, and random epistatic patterns among bits in all objectives $\left(D_{1}, \cdots, D_{M}=\right.$ random $)$. Results presented below show the average performance of the algorithms on 50 different problems randomly generated for each combination of $M, N$ and $K$. That is, in this study 3.500 different problems are used, grouped in subclasses according to the number of epistatic interactions $K$ and number of objectives $M$. In the plots, error bars show $95 \%$ confidence intervals on the mean.

All algorithms used in our study are set with overall parent and offspring populations of size $|\mathcal{P}|=|\mathcal{Q}|=100$, two point crossover for recombination with rate $p_{c}=0.6$, and bit flipping mutation with rate $p_{m}=1 / N$ per bit. The number of evaluations is set to $3 \times 10^{5}$.

We study the performance of $\epsilon$ R-EMO setting the number of subspaces to $N_{S}=\{1,2\}$, varying the parameter $\alpha$. For $N_{S}=1$, no subspace partitioning $\Psi_{N_{S}}=\left\{\psi_{1}=\phi\right\}$, we set $\alpha=\{1.0,0.7,0.5,0.3\}$ so that the desired number of solutions with rank-1 after subspace- $\epsilon$-ranking is $\alpha \times$ $|\mathcal{P}|=\{100,70,50,30\}$, respectively. For $N_{S}=2$, subspace partition $\Psi_{N_{S}}=\left\{\psi_{1}, \psi_{2}\right\}$, we set $\alpha=\{0.5,0.35$, $0.25,0.15\}$ so that $\alpha \times|\mathcal{P}|=\{50,35,25,15\}$, respectively, in each of the two subspaces. Also, note that for $N_{S}=$ 2 the size of the parent population in each subspaces is $\left|P_{s}\right|=|P| / N_{S}$, as indicated in Procedure 1.

For the step of adaptation $\Delta_{s}$, we set its initial value to $\Delta_{0}=0.005(0.5 \%)$ and its maximum and minimum values to $\Delta_{\max }=0.05(5 \%)$ and $\Delta_{\min }=0.0001(0.01 \%)$, respectively.

\section{Effects of Space Partitioning}

\section{1 $\in$ R-EMO with no objective space partitioning}

In this section, we discuss the performance of $\epsilon$ R-EMO when no objective space partitioning is considered $\left(N_{S}=\right.$ 1 ), setting the fraction between desired number of rank1 individuals and population size to $\alpha=\alpha^{*}$ that achieves maximum hypervolume $\mathcal{H}$. Figure 3 (a) shows the average ratio $\frac{\mathcal{H}(E)}{\mathcal{H}(N)}$, where $E$ and $N$ denote the set of solutions found by $\epsilon$ R-EMO and conventional NSGA-II, respectively. Thus, a ratio greater than 1.0 indicates better $\mathcal{H}$ by $\epsilon$ R-EMO than conventional NSGA-II. As a reference, we include a horizontal line to represent the $\mathcal{H}(N)$ values normalized to 1.0. From this figure, we can see that $\epsilon \mathrm{R}$-EMO can significantly improve $\mathcal{H}$ on $4 \leq M \leq 10$ objectives problems, for all values of $K$ (up to $27 \%$ improvement). Note that improvements on $\mathcal{H}$ become larger as we increase the number of objectives $M$ from 4 to 6 , whereas improvements on $\mathcal{H}$ are similarly high for $8 \leq$ $M \leq 10$. Due to space limitations, we include results for $M=\{4,6,8,10\}$ only and not for $M=\{5,7,9\}$.

Improvements on $\mathcal{H}$ can be due to solutions with better convergence, better diversity, or both. To complement 


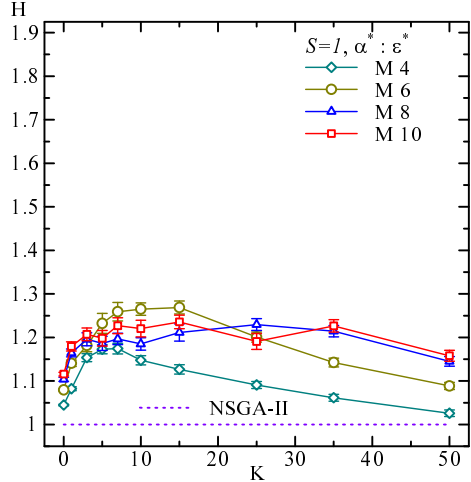

a) Normalized $\mathcal{H}$

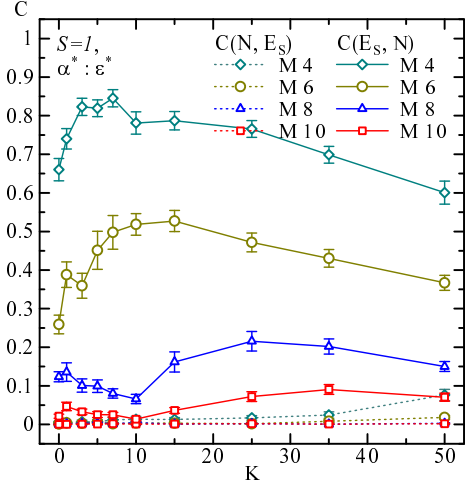

b) $\mathcal{C}$ measure

Fig. 3 Normalized $\mathcal{H}$ and $\mathcal{C}$ between NSGA-II and $\epsilon$ R-EMO when no objective space division $(S=1)$ is considered. $\epsilon \mathrm{R}-\mathrm{EMO}$ is set to $\alpha^{*}$ that achieves maximum $\mathcal{H}(E)$.

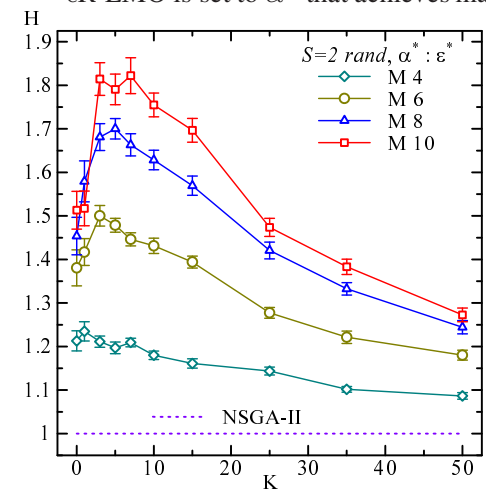

a) Normalized $\mathcal{H}$

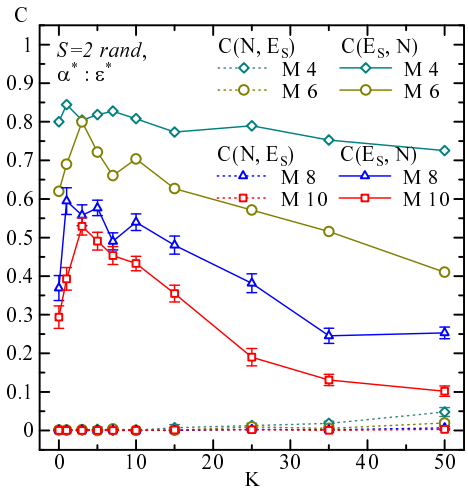

b) $\mathcal{C}$ measure

Fig. 4 Results by NSGA-II and $\epsilon$ R-EMO partitioning the objective space in two subspaces $(S=2)$ using random strategy and setting $\alpha^{*}$ that achieves maximum $\mathcal{H}(E)$.

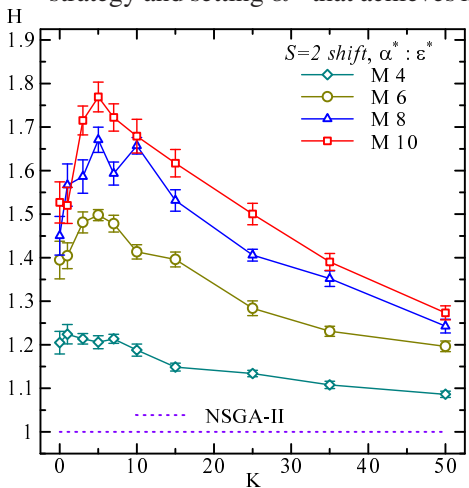

a) Normalized $\mathcal{H}$

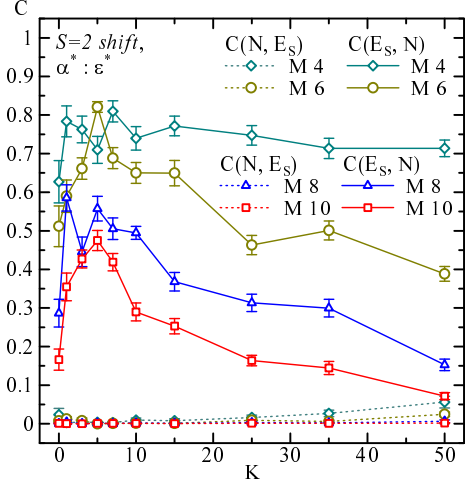

b) $\mathcal{C}$ measure

Fig. 5 Results by NSGA-II and $\epsilon$ R-EMO partitioning the objective space in two subspaces $(S=2)$ using shift strategy and setting $\alpha^{*}$ that achieves maximum $\mathcal{H}(E)$.

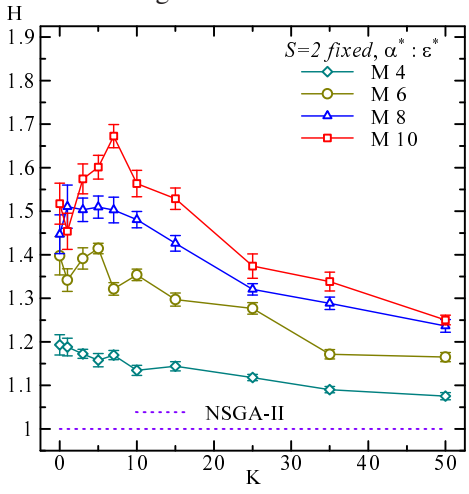

a) Normalized $\mathcal{H}$

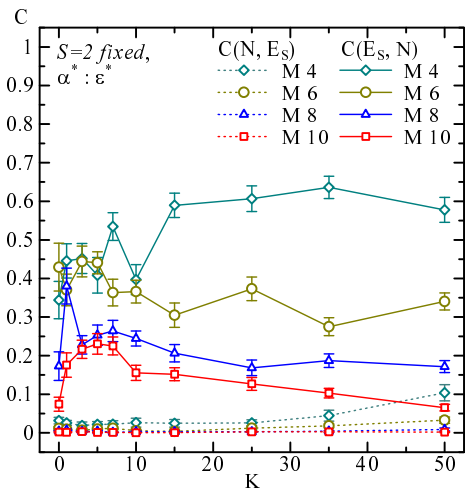

b) $\mathcal{C}$ measure

Fig. 6 Results by NSGA-II and $\epsilon$ R-EMO partitioning the objective space in two subspaces $(S=2)$ using fixed strategy and setting $\alpha^{*}$ that achieves maximum $\mathcal{H}(E)$. 
the analyzes of results on $\mathcal{H}$ we also present results using the $\mathcal{C}$ measure. Figure 3 (b) shows the average $\mathcal{C}$ values between conventional NSGA-II and $\epsilon$ R-EMO set with $\alpha^{*}$. From this figure, we can be see that $\mathcal{C}(N, E)$ is close to 0.0 for most $K$ and $M$. This indicates that there are almost no solutions by conventional NSGA-II that dominate solutions by $\epsilon$ R-EMO. On the other hand, the values of $\mathcal{C}(E, N)$ are very high for 4 objectives (in the range 0.60 $0.85)$ and reduce gradually as we increase $M$ up to $10 \mathrm{ob}-$ jectives (in the range 0.01-0.08). This suggests that a better convergence of solutions contributes to the increases of $\mathcal{H}$ by $\epsilon \mathrm{R}-\mathrm{EMO}$ on $M=4$ problems. As we increase $M$, gains on diversity gradually become more significant than gains on convergence as the reason for the significant improvement of $\mathcal{H}$ on $6 \leq M \leq 10$.

\section{2 $\quad$ R-EMO with objective space partitioning}

In this section we analyze the performance of $\epsilon$ R-EMO partitioning instantaneously the objective space into two subspaces using the random, shift, and fixed partition strategies introduced in section Chapter 3.

First, we show results by the random partitioning strategy in Figure 4. Looking at Figure 4 (a) and comparing with Figure 3 (a), we can see that ranking on subspaces using a random strategy leads to a remarkable improvement on $\mathcal{H}$ for all values of $M$ and $K$ (up to $82.5 \%$ improvement). Note that the increase on $\mathcal{H}$ gets bigger with the number of objectives $M$. Looking at at Figure 4 (b) and comparing with Figure 3 (b), we can see that $\mathcal{C}(E, N)$ also increases for any value of $K$ and $M$, whereas $\mathcal{C}(N, E)$ remains close to zero. That is, convergence also improves substantially.

Next, we discuss results by the shift partition strategy shown in Figure 5 (a) and (b). From these figures note that the shift strategy also leads to a remarkable improvement on $\mathcal{H}$ and $\mathcal{C}$. Comparing the shift and random strategies, the latter leads to slightly better results than the former especially for $M \geq 8$ and $K \leq 15$. As mentioned above, the random strategy can sample any possible subspace of $\phi$, whereas the shift strategy can sample only $M$ of all possible subspaces of $\phi$. The number of subspaces unable to sample the shift strategy increase with the dimension of the objective space, especially if we keep constant the number of subspaces. Better results by the random strategy suggest that sampling more subspaces becomes relevant as the number of objectives increase. However, since results by the random and shift strategies are not very different, this also suggests that not all subspaces need to be sampled to achieve good performance, which could be highly relevant to dimensionality reduction and parallelization under a space partitioning approach.

Results by the fixed partition strategy are shown in Figure 6 (a) and (b). See that the fixed strategy leads to smaller $\mathcal{H}$ and $\mathcal{C}(E, N)$ than the random and shift partition strategies. Comparing to $\epsilon \mathrm{R}-\mathrm{EMO}$ with no subspace partitioning, the fixed strategy leads to higher $\mathcal{H}$ on all $M$ but with smaller $\mathcal{C}(E, N)$ on $M \leq 6$. We did not expect to achieve best performance with the fixed strategy. However, since this strategy only explores two of all possible subspaces of $\phi$, these results are rather surprising and illustrate the gains in performance that could be achieved in high-dimensional spaces by space partitioning even if poor partition strategies are used.

It should be noted that as we increase $K$ (non-linearity of the problem) improvements on both $\mathcal{H}$ and $\mathcal{C}$ reduce regardless of the partition strategy. This suggests that in addition to better selection strategies, we should also look into ways to improve recombination and mutation to achieve better performance on highly non-linear problems.

The timing to reassign objectives to subspaces is an important aspect that could lead to better performance and facilitate parallel implementations of the proposed method. The fixed and random strategies explored in this work are two extremes scenarios between no reassignment at all and reassignment at each generation, which could be seen as exploitation within a subspace versus exploration through different subspaces, respectively. A reassignment of objectives to subspaces after a certain number of generations may lead to a better balance between these two important issues in selection. This is an important line of research that we would like to pursue in the future.

\section{$6 \cdot 3$ Analysis of $\alpha$}

In this section we analyze the parameter $\alpha$ that determines the desired number of rank-1 solution in each subspace. As an example, Figure 7 shows $\mathcal{H}$ and $\mathcal{C}$ results achieved by different settings of $\alpha$ on $M=8$ objectives landscapes partitioning the objective space in $N_{S}=2$ subspaces using shift strategy. Note that, since we use an overall parent population size of $|P|=100$, the size of the parent population in each one of the two subspaces is $\left|P_{s}\right|=|P| / N_{S}=50$. Also, recall that the number of rank1 solutions in each subspace is calculated as a fraction of the overall parent population by $\alpha \times|P|$.

From Figure 7, note that $\alpha \geq 0.25$ (at least $50 \%$ of the parent population $P_{s}$ in each subspace is given rank-1) leads to high performance, whereas results by $\alpha=0.15$ are clearly lower on $M=8$ objectives landscapes. Analyzing performance by $\alpha \geq 0.25$, see that setting $\alpha$ to 0.35 or 0.25 leads to best performance for most $K$, both on $\mathcal{H}$ 


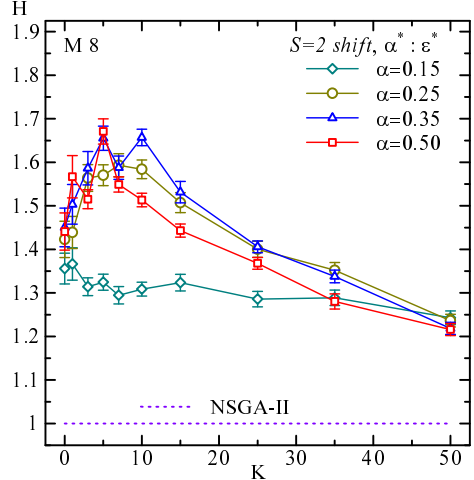

a) Normalized $\mathcal{H}$

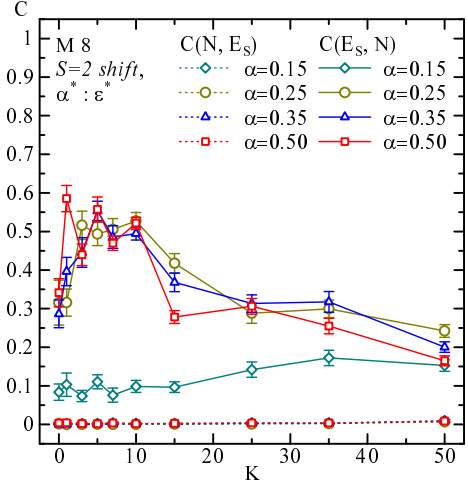

b) $\mathcal{C}$ measure

Fig. 7 Results by NSGA-II and $\epsilon$ R-EMO partitioning the objective space in two subspaces $(S=2)$ using shift strategy and varying $\alpha$ on $M=8$ objectives.

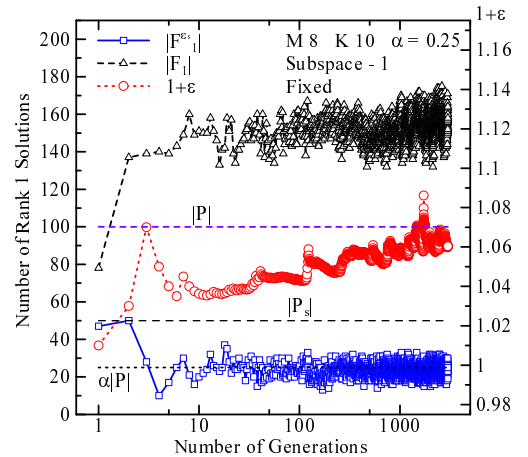

(a) Fixed

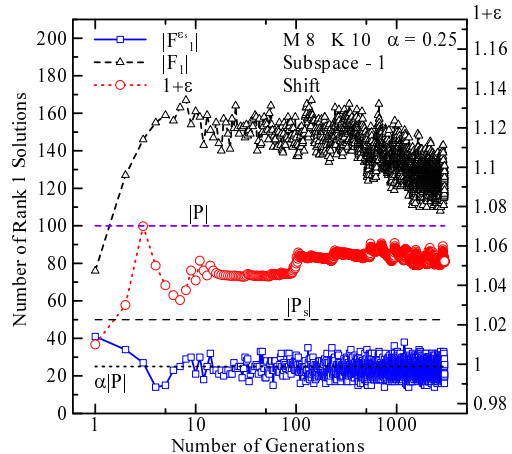

(b) Shift

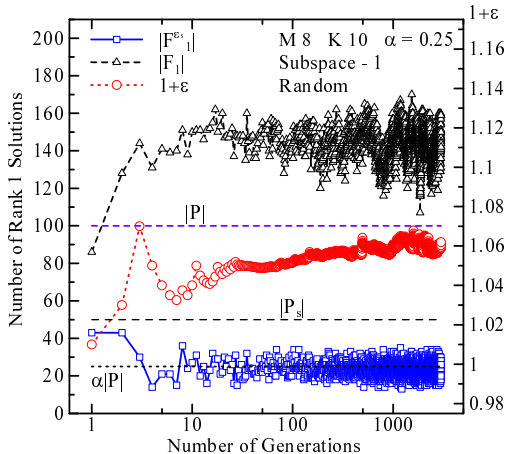

(c) Random

Fig. 8 Adaptation of $\epsilon$ in $\epsilon$ R-EMO by fixed, shift, and random partitioning strategies for $\alpha=0.25$ on $M=8$ objectives and $K=10$ epistatic bits.

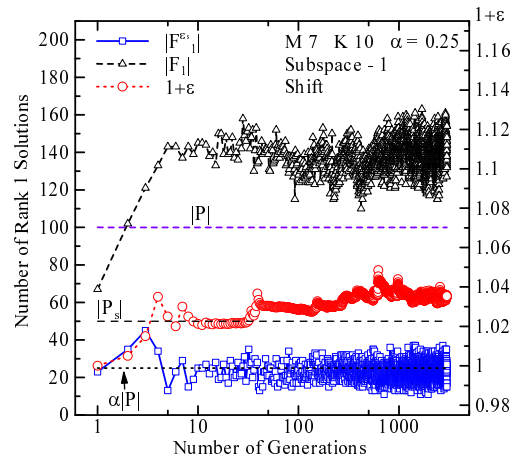

(a) Three-dimensional subspace

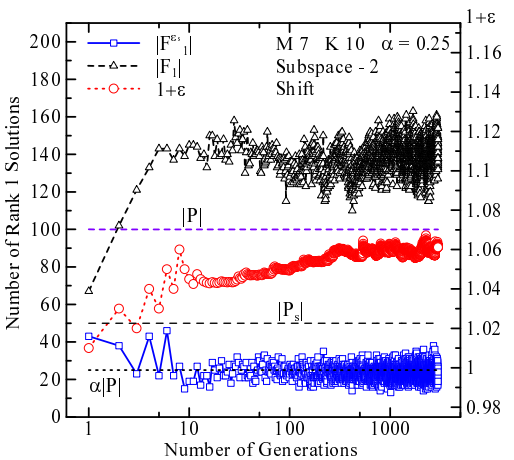

(b) Four-dimensional subspace

Fig. 9 Concurrent adaptation of $\epsilon$ on subspaces of different dimensionality by $\epsilon$ R-EMO with shift partitioning strategy for $\alpha=0.25$ on $M=7$ objectives and $K=10$ epistatic bits.

and $\mathcal{C}$. However, see that setting $\alpha$ to 0.5 could give highest performance especially on small $K$. Although there is not an absolute winner among $\alpha \geq 0.25$ values, it is important to note that subspace partitioning's lower bound performance $(\min \mathcal{H} \wedge \min \mathcal{C}(E, N), \forall \alpha \in\{0.5,0.35,0.25\})$ is significantly better than the performance by no subspace partitioning (see Figure 7 and compare with Figure 3, $M=8$ ). Analyzing our data for other values of $M$, in general, we see that performance by $\alpha=0.25$ is better than 0.35 when the number of objectives decrease to $M=6$ and $M=4$; whereas performance by 0.5 and 0.35 is better than 0.25 when we increase $M$ to 10 objectives. As a rule of thumb, when the space is partitioned into 2 subspaces, $\alpha=0.25$ works well on $M=4$ and $M=6, \alpha=0.35$ on $M=8$, and $\alpha=0.5$ on $M=10$.

\subsection{Adaptation of $\epsilon$}

In this section we discuss the adaptation of $\epsilon_{s}$. Figure 8 (a)-(c) illustrate the adaptation of $\epsilon_{s}$ in one of the two four-dimensional subspaces by fixed, shift, and random partitioning strategies for $\alpha=0.25$ in a $M=8$ and $K=10$ landscape. These figures depict results of a single run of the algorithm. The horizontal lines indicate the size of the overall parent population $|\mathcal{P}|=100$, the size of 
the parent population in the subspace $\left|\mathcal{P}_{s}\right|=50$, and the desired number of rank-1 individuals in the subspace $\alpha \times$ $|\mathcal{P}|=25$. From these figures note that the number of nondominated individuals $\left|\mathcal{F}_{1}\right|$ (considering all objectives) exceeds $|\mathcal{P}|$ since the initial generations, as shown by the line with triangles. See also that the adaptive mechanism appropriately varies $\epsilon_{s}$ throughout the generations, as illustrated by the line with circles, so that after subspace- $\epsilon$ ranking the number of rank-1 individuals $\left|\mathcal{F}_{1}^{\epsilon_{s}}\right|$ in the subspace is kept around the desired number $\alpha \times|\mathcal{P}|$ as shown by the line with squares. Note that adaptation works appropriately regardless of the partition strategy.

Note that we adapt a different $\epsilon_{s}$ for each subspace in order to have a general adaptive algorithm that can search concurrently on subspaces of different dimensionality, where very dissimilar distributions of solutions appear. Figure 9 (a) and (b) show the concurrent adaptation of $\epsilon_{s}$ in the three and four-dimensional subspaces, respectively, obtained by partitioning a $M=7$ objectives landscapes into two subspaces using shift strategy. From the figures note that very different values of $\epsilon$ are required for subspaces of different dimensionality in order to keep the number of rank-1 individuals around the desired number.

When the concurrent search is performed on subspaces of the same dimensionality, the adaptation trend of $\epsilon$ for each subspace is similar suggesting that in this particular case one $\epsilon$ could be adequate for all subspaces. However, this deserves further investigation. We should recall that in this work landscapes of similar non-linear complexity are used in all objectives (given by the number of epistatic interaction $K_{1}=K_{2}=\cdots=K_{M}=K$ ). The complexity of the landscapes associated to each objective function also affects the distribution of solutions [Aguirre 06], which could lead to dissimilar adaptation trends of $\epsilon$ even if subspaces of the same dimensionality are used.

\section{Comparison with Substitute Assignment Distance Methods}

In this section we compare the performance among objective space partitioning with adaptive $\epsilon$-ranking $\epsilon$ R-EMO and two substitute distance assignment methods, namely Subvector Dominance Assignment (SVDOM) and Epsilon Dominance Assignment (EPSDOM) [Koppen 07]. These three many-objective methods enhance selection using different strategies to recalculate the primary or secondary ranking of solutions. As explained before, adaptive $\epsilon$ ranking in $\epsilon$ R-EMO focuses on the primary ranking of solutions. On the other hand, SVDOM and EPSDOM focus on the secondary ranking of solutions, replacing the diver- sity estimator with a substitute assignment distance. The motivation for this comparison is to understand better the effects on performance of different strategies that re-rank solutions to improve Pareto selection of many-objective optimizers.

The substitute distance that determines the secondary ranking of solutions in SVDOM and EPSDOM are based on measurement procedures that calculate the highest degree to which a solution is nearly Pareto dominated by any other solution [Koppen 07]. SVDOM re-ranks a nondominated solution based on the number of objectives that are better in other non-dominated solutions. On the other hand, EPSDOM re-ranks a non-dominated solution based on the magnitude that other non-dominated solutions need to improve in order to dominate it. The reader is referred to [Koppen 07] for details on SVDOM and EPSDOM.

Figure 10 (a)-(c) show the average hypervolume ratios between the many-objective algorithms and conventional NSGA-II. That is, $\frac{\mathcal{H}(S)}{\mathcal{H}(N)}, \frac{\mathcal{H}(P)}{\mathcal{H}(N)}$, and $\frac{\mathcal{H}(E)}{\mathcal{H}(N)}$, where $S, P$, $E$, and $N$ denote the set of solutions found by SVDOM, EPSDOM, $\epsilon$ R-EMO and conventional NSGA-II, respectively. From these figures, it can be seen that $\epsilon$ R-EMO achieves better hypervolume than EPSDOM and SVDOM for all $M$ and $K$. Also note that between the substitute distance assignment algorithms, EPSDOM achieves better hypervolume than SVDOM for all $M$ and $K$. Note that in this work we use $(0.0,0.0, \cdots, 0.0)$ as reference point to calculate the hypervolume. This point is far away from the Pareto front found by the algorithms and would relatively emphasize solutions located in the edges of the multi-dimensional Pareto front in the calculation of the hypervolume.

Figure 11 (a)-(c) show the average $\mathcal{C}$ values between conventional NSGA-II and the many-objective algorithms. From these figures, it can be seen that $\mathcal{C}$ values by EPSDOM are better than SVDOM for most $M$ and $K$. Also, note that $\mathcal{C}$ by $\epsilon \mathrm{R}-\mathrm{EMO}$ is better than SVDOM for $M=$ $\{4,6\}$, but slightly worse for $M=\{8,10\}$. Comparing EPSDOM and $\epsilon$ R-EMO, overall EPSDOM achieves similar or better $\mathcal{C}$ than $\epsilon$ R-EMO.

The above results on $\mathcal{H}$ and $\mathcal{C}$, i.e. better $\mathcal{H}$ by $\epsilon \mathrm{R}$ EMO and a better $\mathcal{C}$ by EPSDOM, suggest that there is a diversity-convergence trade-off between $\epsilon$ R-EMO and EPSDOM. Figure 12 (a)-(d) show $\mathcal{C}$ values between $\epsilon$ REMO and EPSDOM. From these figures, note that some solutions by $\epsilon$ R-EMO are dominated by solutions of EPSDOM, especially for $M \geq 6$. These results, confirm that convergence is better by EPSDOM than $\epsilon$ R-EMO. However, note that the fraction of dominated solutions in $\epsilon \mathrm{R}$ EMO is larger for $M=6$ and reduces with the number of 


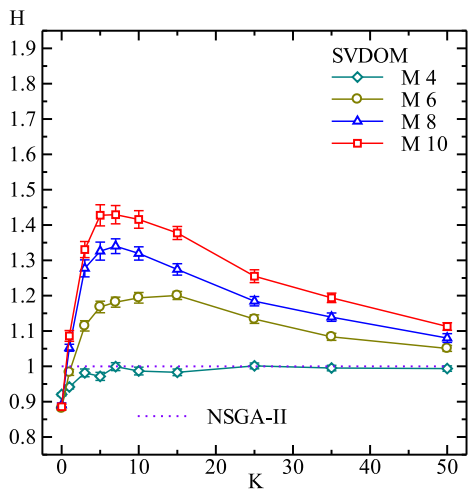

(a) SVDOM

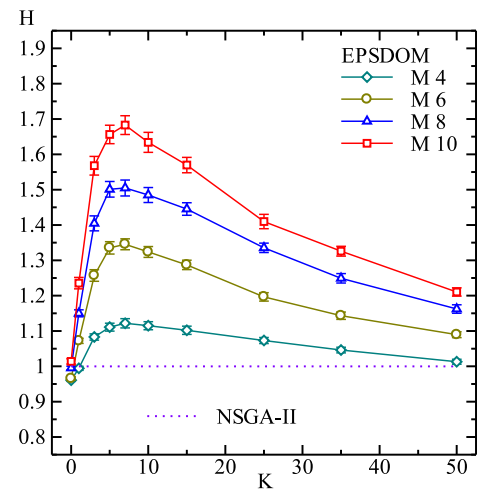

(b) EPSDOM

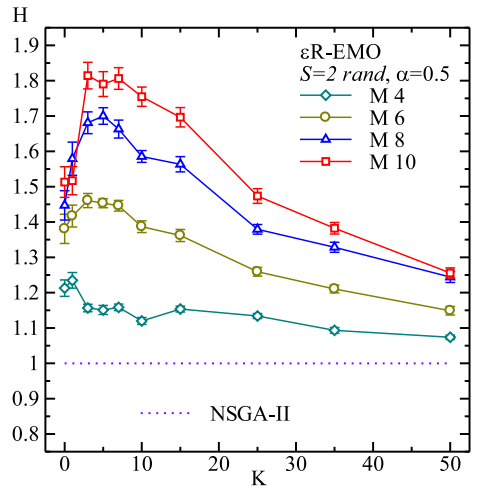

(c) $\epsilon$ R-EMO

Fig. 10 Normalized $\mathcal{H}$ by SVDOM, EPSDOM, and $\epsilon$ R-EMO. $M=\{4,6,8,10\}$ objectives.

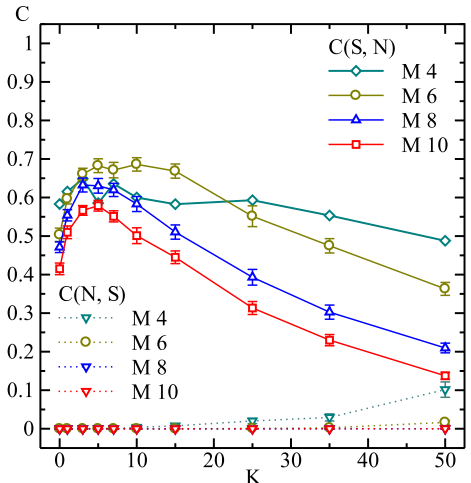

(a) SVDOM

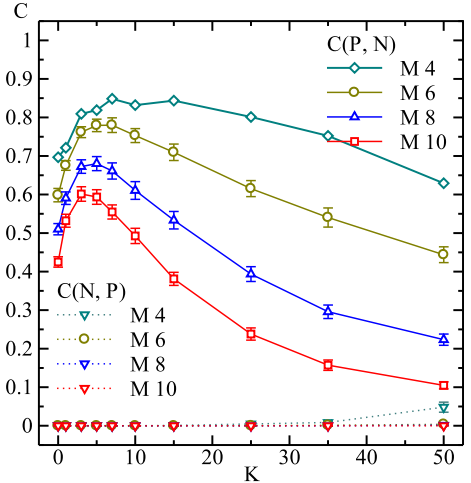

(b) EPSDOM

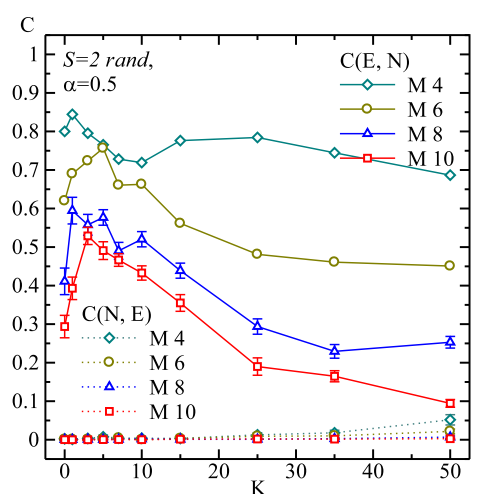

(c) $\epsilon$ R-EMO

Fig. $11 \mathcal{C}$ values by SVDOM, EPSDOM, and $\epsilon$ R-EMO. $M=\{4,6,8,10\}$ objectives.

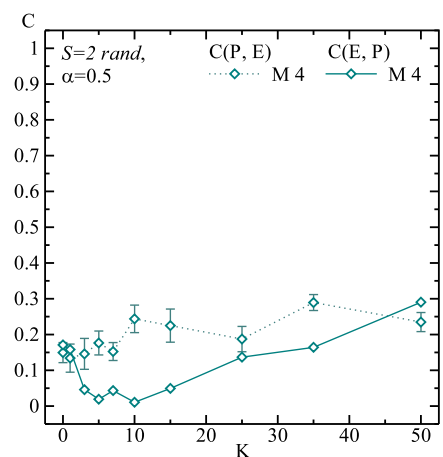

(a) $M=4$

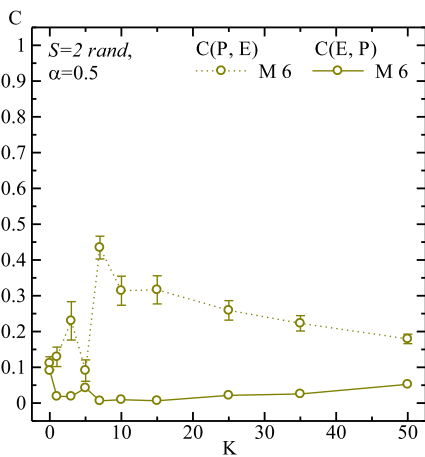

(b) $M=6$

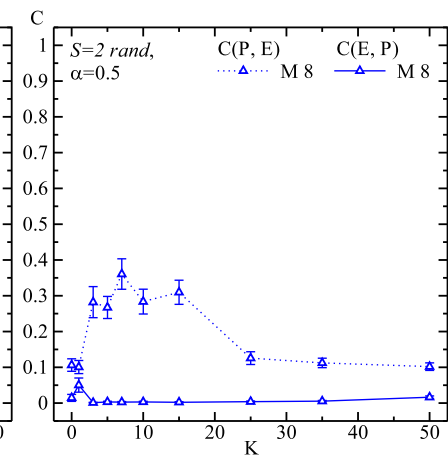

(c) $M=8$

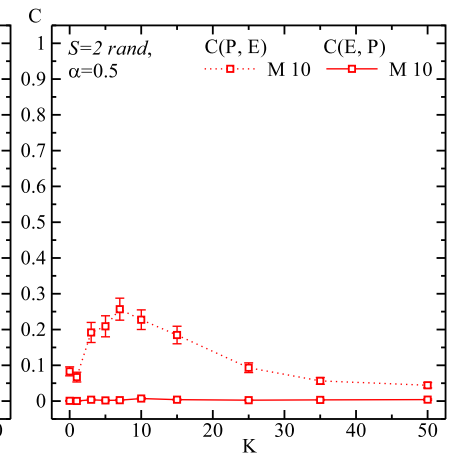

(d) $M=10$

Fig. $12 \mathcal{C}$ values between EPSDOM and $\epsilon$ R-EMO. $M=\{4,6,8,10\}$ objectives.

objectives.

Figure 13 (a)-(c) show the normalized maximum spread $\mathcal{D}$ by SVDOM, EPSDOM and $\epsilon$ R-EMO. From these figures, note that $\mathcal{D}$ is substantially better by $\epsilon$ R-EMO than EPSDOM and SVDOM for all $M$ and $K$; whereas $\mathcal{D}$ is slightly better by EPSDOM than by SVDOM. These results show that $\epsilon$ R-EMO can find solutions in a region of objective space broader than the region where substitute assignment distance algorithms find solutions.

Figure 14 (a)-(d) plot the maximum attained fitness values in each objective function by conventional NSGA-II, $\epsilon$ R-EMO, SVDOM, and EPSDOM for $M=\{4,6,8,10\}$ objectives and $K=7$ epistatic interactions. Note that SVDOM and EPSDOM attain high values of $\mathcal{H}$ and $\mathcal{C}$ at $K=$
7. From Figure 14, we can see that conventional NSGAII achieves in all objectives better maximum-fitness values than SVDOM and EPSDOM. Note also that the gap between NSGAII and SVDOM/ EPSDOM increases with the number of objectives $M$ of the problem. These results confirm that solutions found by SVDOM and EPSDOM tend to concentrate within an increasingly reduced space of objective space as the number of objectives of the problem increase. On the other hand, note that $\epsilon \mathrm{R}-\mathrm{EMO}$ obtains overall better maximum-fitness than conventional NSGA-II in all $M$. These results show that the good values of hypervolume by SVDOM and EPSDOM are exclusively due to a better convergence of some solutions in a narrow region of objective space at the expense of diver- 


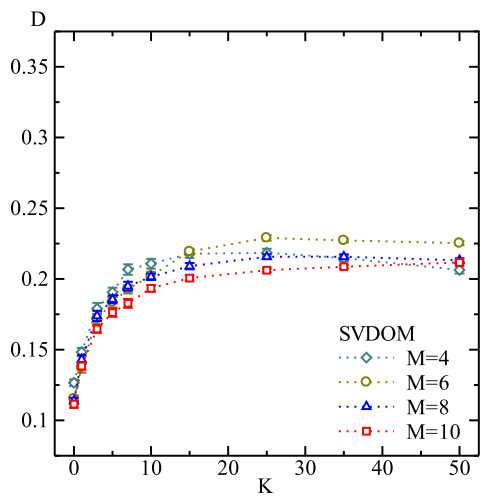

(a) SVDOM

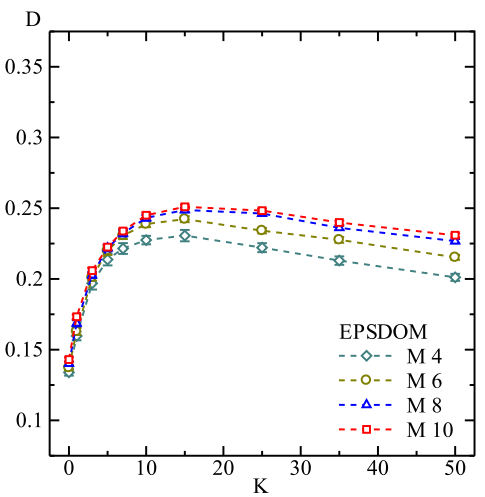

(b) EPSDOM

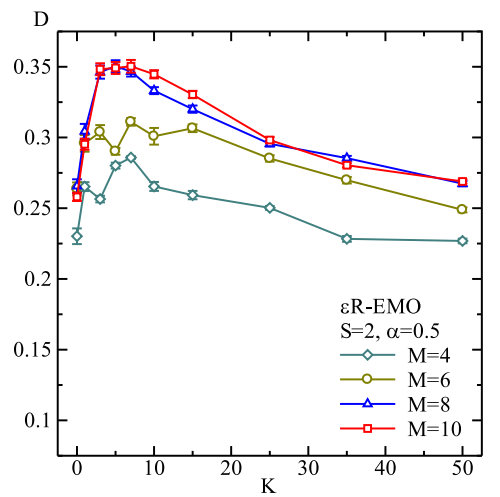

(c) $\epsilon$ R-EMO

Fig. 13 Normalized maximum spread $\mathcal{D}$ by SVDOM, EPSDOM, and $\epsilon$ R-EMO. $M=\{4,6,8,10\}$ objectives.

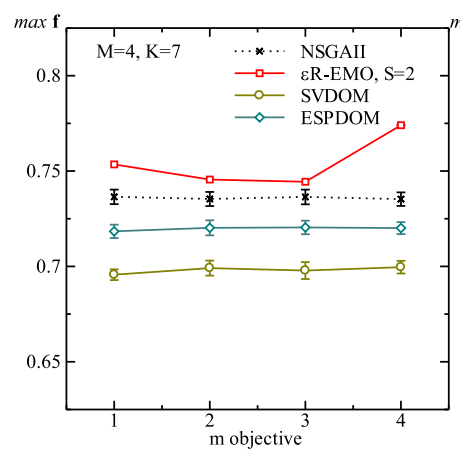

(a) $M=4, K=7$

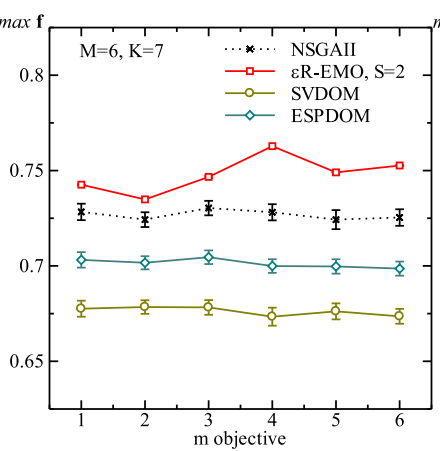

(b) $M=6, K=7$

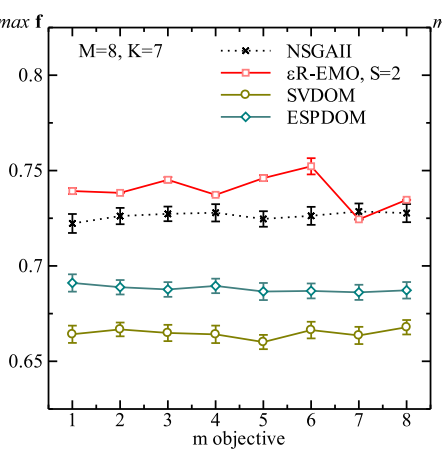

(c) $M=8, K=7$

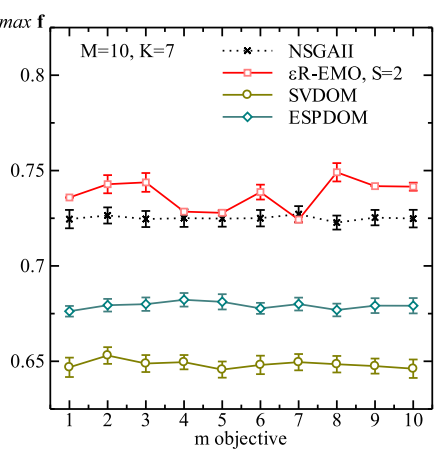

(d) $M=10, K=7$

Fig. 14 Maximum achieved fitness in each objective by NSGA-II, SVDOM, EPSDOM, and $\epsilon$ R-EMO. $M=$ $\{4,6,8,10\}$ objectives and $K=7$.

sity. These results show that adaptive $\epsilon$-ranking and substitute distance assignment EPSDOM emphasize different areas of objective space, suggesting that both strategies could complement each other to produce better approximations of the Pareto front.

\section{Conclusions}

This work has proposed space partitioning for evolutionary many-objective optimization focusing on improving selection of the optimizer. We implemented space partitioning using adaptive $\epsilon$-ranking showing that the performance of a many-objective optimizer can be improved considerable, in terms of both convergence and diversity of solutions, by instantaneously partitioning the objective space into subspaces and concurrently searching within each subspace. Also, we analyzed the relevance of the partition strategy used to define a schedule of subspace sampling in order to emphasize different subspaces at different generations of the algorithm. We showed that a strategy able to sample all possible subspaces, without seeking to keep any correlation between subspaces from one generation to the next, gives better performance than strategies that emphasize the correlation of the subspaces at the expense of not being able to sample all subspaces. In addition, we compared the space partitioning algorithm with two substitute distance assignment many-objective optimizers; namely SVDOM and EPSDOM. The three manyobjective optimizers use different strategies to enhance the effectiveness of selection in high-dimensional spaces. We showed that the adaptive $\epsilon$-ranking strategy and the substitute distance assignment strategies emphasize different areas of objective space and that could be used as complementary strategies to produce a better approximation of the high dimensional Pareto front.

As future works, we would like to extend the space partitioning approach to apply complementary ranking strategies concurrently, include dynamic dimensionality reduction, and realize parallel implementations of many-objective optimizers.

\section{Acknowledgments}

This study was performed through Special Coordination Funds for Promoting Science and Technology of the Ministry of Education, Culture, Sports, Science and Technology, of the Japanese Government.

\section{$\diamond$ References $\diamond$}

[Aguirre 04] H. Aguirre and K. Tanaka, "Insights on Properties of Multiobjective MNK-Landscapes", Proc. 2004 IEEE Congress on Evolutionary Computation, IEEE Service Center, pp.196-203, 2004. [Aguirre 05] H. Aguirre and K. Tanaka, "Selection, Drift, Recombination, and Mutation in Multiobjective Evolutionary Algorithms 
on Scalable MNK-Landscapes", Proc. Third International Conference on Evolutionary Multi-Criterion Optimization (EMO 2005), Springer, LNCS, vol. 3410, pp. 355-369, 2005.

[Aguirre 06] H. Aguirre and K. Tanaka, "Working Principles, Behavior, and Performance of MOEAs on MNK-Landscapes", European Journal of Operational Research, Elsevier, vol. 181(3), pp. 16701690, September 2007.

[Aguirre 08] H. Aguirre and K. Tanaka, "Robust Optimization by $\epsilon$ Ranking On High Dimensional Objective Spaces", Proc. 7th Intl. Conf. on Simulated Evolution and Learning (SEAL 2008), Springer, LNCS, vol. 5361, pp. 421-431, 2008.

[Aguirre 09a] H. Aguirre and K. Tanaka, "Adaptive $\epsilon$-Ranking on MNK-Landscapes", Proc. 2009 IEEE Symposium on Computational Intelligence in Multicriteria Decision Making, 2009.

[Aguirre 09b] H. Aguirre and K. Tanaka, "Many-Objective Optimization by Space Partitioning and Adaptive $\epsilon$-Ranking on MNKLandscapes", Proc. 5th Intl. Conf. on Evolutionary Multi-Criterion Optimization, Springer, LNCS vol.5467, pp.407-422, 2009.

[Aguirre 09c] H. Aguirre, K. Tanaka, "Space Partitioning with Adaptive $\epsilon$-Ranking and Substitute Distance Assignments: A Comparative Study on Many-Objective MNK-Landscapes", Proc. 2009 Genetic and Evolutionary Computation Conference, ACM Press, pp. 547-554, July 2009.

[Branke 04] J. Branke, H. Schmeck, K. Deb and M. Reddy, "Parallelizing Multi-Objective Evolutionary Algorithms: Cone Separation ", Proc. Congress on Evolutionary Computation (CEC 2004), IEEE Press , pp.1952-1957, 2004.

[Brockhoff 07] D. Brockhoff and E. Zitzler,"Are All Objectives Necessary? On Dimensionality Reduction in Evolutionary Multiobjective Optimization," Parallel Problem Solving from Nature - PPSN IX, LNCS (Springer), vol. 4193, pp. 533-542, 2006.

[Coello 02] C. Coello, D. Van Veldhuizen, and G. Lamont, Evolutionary Algorithms for Solving Multi-Objective Problems. Kluwer Academic Publishers, Boston, 2002.

[Corne 07] D. Corne and J. Knowles, "Techniques for Highly Multiobjective Optimization: Some Non-dominated Points are Better than Others," Proc.of 2007 Genetic and Evolutionary Computation Conference (GECCO 2007)", pp.773-780, 2007.

[Deb 00] K. Deb, S. Agrawal, A. Pratap and T. Meyarivan, "A Fast Elitist Non-Dominated Sorting Genetic Algorithm for MultiObjective Optimization: NSGA-II", Kan GAL report 200001, 2000.

[Deb 01] K. Deb, Multi-Objective Optimization using Evolutionary Algorithms, John Wiley \& Sons, Chichester, West Sussex, England, 2001.

[Deb 06a] K. Deb and J. Sundar,"Preference Point Based Multiobjective Optimization Using Evolutionary Algorithms," Proc. of 2006 Genetic and Evolutionary Computation Conference (GECCO 2006), pp. 635-642, 2006.

[Deb 06b] K. Deb and K. Saxena,"Searching for Pareto-optimal Solutions Through Dimensionality Reduction for Certain Largedimensional Multi-objective Optimization Problems," Proc. of 2006 IEEE Congress on Evolutionary Computation (CEC 2006), pp. 3353 3360,2006 .

[Emmerich 05] M. Emmerich, N. Beume, and B. Naujoks, "An EMO Algorithm Using the Hypervolume Measure as Selection Criterion", Proc. 3rd Intl. Conf. on Evolutionary Multi-Criterion Optimization, LNCS (Springer), vol. 3410, pp. 62-76, 2005.

[Fonseca 06] C. Fonseca, L. Paquete, and M. López-Ibáñez, "An Improved Dimension-sweep Algorithm for the Hypervolume Indicator", Proc. 2006 IEEE Congress on Evolutionary Computation, IEEE Service Center, pp.1157-1163, 2006

[Hughes 05] E. J. Hughes, "Evolutionary Many-Objective Optimization: Many Once or One Many?", Proc. 2005 IEEE Congress on Evolutionary Computation, IEEE Service Center, Vol.1, pp.222-227, September 2005.

[Ishibuchi 07a] H. Ishibuchi and Y. Nojima, "Optimization of Scalarizing Functions Through Evolutionary Multiobjective Optimization," Proc. Fourth Int. Conf. on Evolutionary Multi-Criterion Optimization (EMO 2007), LNCS (Springer), vol. 4403, pp. 51-65, 2007.

[Ishibuchi 07b] H. Ishibuchi, N. Tsukamoto, and Y. Nojima, "Iterative Approach to Indicator-based Multiobjective Optimization," Proc. of
2007 IEEE Congress on Evolutionary Computation (CEC 2007), pp. 3697-3704, 2007.

[Ishibuchi 08] H. Ishibuchi, N. Tsukamoto, and Y. Nojima, "Evolutionary Many-Objective Optimization: A Short Review", In Proc. IEEE Congress on Evolutionary Computation (CEC 2008), IEEE Press, pp.2424-2431, 2008.

[Kauffman 93] S. A. Kauffman, The Origins of Order: SelfOrganization and Selection in Evolution, Oxford University Press, 1993.

[Koppen 07] M.Koppen and K. Yoshida, "Substitute Distance Assignments in NSGA-II for Handling Many-Objective Optimization Problems", Proc. 4th Intl. Conf. on Evolutionary Multi-Criterion Optimization, Springer, LNCS, vol.4403, pp,727-741, 2007.

[Kukkonen 07] S. Kukkonen and J. Lampinen, "Ranking-dominance and Many Objective Optimization," Proc. of 2007 IEEE Congress on Evolutionary Computation (CEC 2007), pp. 3983-3990, 2007.

[Laumanns 02] M. Laumanns, L. Thiele, K. Deb and E. Zitzler, "Combining Convergence and Diversity in Evolutionary Multiobjective Optimization", Evolutionary Computation, Vol.10, No.3, pp.263-282, Fall 2002.

[López Jaimes 08] A. López Jaimes, C. Coello, and D. Chakraborty, "Objective Reduction Using a Feature Selection Technique", Proc. 2008 Genetic and Evolutionary Computation Conference (GECCO 2008), ACM Press, pp.674-680, July 2008.

[Purshouse 03] R. Purshouse and P. Fleming, "Conflict, Harmony, and Independence: Relationships in Evolutionary Multi-criterion Optimisation", Second Intl. Conf. on Evolutionary Multi-Criterion Optimization, Springer, LNCS, vol. 2632, pp.16-30, April 2003.

[Yu 74] P. L. Yu, "Cone Convexity, Cone Extreme Points, and Nondominated Solutions in Decision Problems with Multiobjectives", Journal of Optimization Theory and Applications, vol.14, no.3, pp. 319-377, 1974.

[Zitzler 99] E. Zitzler, Evolutionary Algorithms for Multiobjective Optimization: Methods and Applications, $\mathrm{PhD}$ thesis, Swiss Federal Institute of Technology, Zurich, 1999.

[Zitzler 04] E. Zitzler and S. Kunzli, "Indicator-based Selection in Multiobjective Search", Proc. 8th Intl. Conf. on Parallel Problem Solving from Nature, Springer, LNCS, vol.3242, pp. 832-842, 2004.

\section{〔担当委員：廣安 知之〕}

Received July 29, 2009.

\section{Author's Profile}

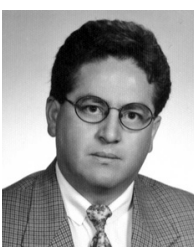

\section{Hernán Aguirre}

received his Engineer degree in computer systems from Escuela Politécnica Nacional, Quito, Ecuador in 1992. From 1997 to 2003 he was a research scholar sponsored by the Japanese Ministry of Education, Culture, Sports, Science and Technology. He received the M.S. and Ph.D. degrees from Shinshu University, Japan, in 2000 and 2003, respectively. Currently, he is an assistant professor at Shinshu University. His research interests include evolutionary computation, multidisciplinary design optimization, computational intelligence, information security, parallel computing, and their applications. He is a member of IEEE, ACM-SIGEVO, and IPSJ.

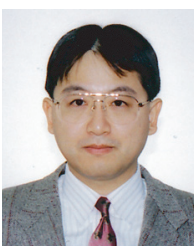

\section{Kiyoshi Tanaka}

received B.S and M.S. degrees in Electrical Engineering and Operations Research from National Defense Academy, Yokosuka, Japan, in 1984 and 1989, respectively. In 1992, he received Dr. Eng. degree from Keio University, Tokyo, Japan. In 1995, he joined the Department of Electrical and Electronic Engineering, Faculty of Engineering, Shinshu University, Nagano, Japan, where he is currently a professor. His research interests include image and video processing, information hiding, evolutionary computation, chaos \& fractals, and their applications. He is a member of IEEE, IEICE, IPSJ and IIEEJ. He is the vice-chairman of journal editing committee of IIEEJ. 